\title{
SCHUR-CONVEXITY OF THE COMPLETE ELEMENTARY SYMMETRIC FUNCTION
}

\author{
KAIZHONG GUAN
}

Received 2 October 2004; Revised 15 January 2005; Accepted 27 January 2005

We prove that the complete elementary symmetric function $c_{r}=c_{r}(x)=C_{n}^{[r]}(x)=$ $\sum_{i_{1}+\cdots+i_{n}=r} x_{1}^{i_{1}} \cdots x_{n}^{i_{n}}$ and the function $\phi_{r}(x)=c_{r}(x) / c_{r-1}(x)$ are Schur-convex functions in $R_{+}^{n}=\left\{\left(x_{1}, x_{2}, \ldots, x_{n}\right) \mid x_{i}>0\right\}$, where $i_{1}, i_{2}, \ldots, i_{n}$ are nonnegative integers, $r \in N=\{1$, $2, \ldots\}, i=1,2, \ldots, n$. For which, some inequalities are established by use of the theory of majorization. A problem given by K. V. Menon (Duke Mathematical Journal 35 (1968), $37-45)$ is also solved.

Copyright (c) 2006 Kaizhong Guan. This is an open access article distributed under the Creative Commons Attribution License, which permits unrestricted use, distribution, and reproduction in any medium, provided the original work is properly cited.

\section{Introduction}

Consider the complete elementary symmetric function

$$
c_{r}=c_{r}(x)=C_{n}^{[r]}(x)=\sum_{i_{1}+\cdots+i_{n}=r} x_{1}^{i_{1}} \cdots x_{n}^{i_{n}}
$$

where $i_{1}, i_{2}, \ldots, i_{n}$ are nonnegative integers, $r \in N$. Define $c_{0}(x)=1$. Correspondingly, the generalized $r$-order symmetric mean is

$$
D_{r}(x)=D_{n}^{[r]}(x)=\left(\begin{array}{c}
r+n-1 \\
n-1
\end{array}\right)^{-1} C_{n}^{[r]}(x),
$$

where $\left(\begin{array}{c}r+n-1 \\ n-1\end{array}\right)=(n+r-1) ! /(n-1) ! r !$.

For (1.1) and (1.2), Menon [7] mainly obtained the following results

$$
\begin{gathered}
\left(C_{n}^{[r]}(a+b)\right)^{1 / r} \leq\left(C_{n}^{[r]}(a)\right)^{1 / r}+\left(C_{n}^{[r]}(b)\right)^{1 / r} \\
c_{r}(a) \mathcal{c}_{s-1}(a) \geq c_{r-1}(a) c_{s}(a), \quad 0<r<s
\end{gathered}
$$


2 The complete elementary symmetric function

$$
\begin{gathered}
\left(c_{r}(a)\right)^{1 / r} \geq\left(c_{s}(a)\right)^{1 / s}, \quad 0<r<s ; \\
D_{r-2}(a) D_{r+2}(a)-D_{r-1}(a) D_{r+1}(a) \geq 0, \quad n=2 .
\end{gathered}
$$

When $n>2$, is inequality (1.6) true? This problem was given out by Menon in [7].

Detemple and Robertson [2] derived

$$
D_{r-1}(a) D_{r+1}(a)-D_{r}^{2}(a) \geq 0, \quad r=1,2,3 .
$$

Whether inequality (1.7) is still valid for $r \geq 4$ was given in [5], and this problem was solved in [3].

The Schur-convex functions were introduced by I. Schur in 1923 [6], and has many important applications in analytic inequalities. Hardy et al. were also interested in some inequalities that are related to Schur-convex functions [4], the following definitions can be found in many references such as $[5,6,8,9]$.

Definition 1.1. Suppose that $x_{i}, y_{i} \in R, i=1,2, \ldots, n, x=\left(x_{1}, \ldots, x_{n}\right)$ and $y=\left(y_{1}, \ldots, y_{n}\right)$. Rearrange the components of $x$ and $y$ such that $x_{[1]} \geq x_{[2]} \geq \cdots \geq x_{[n]}, y_{[1]} \geq y_{[2]} \geq$ $\cdots \geq y_{[n]}$. If $\sum_{i=1}^{k} x_{[i]} \leq \sum_{i=1}^{k} y_{[i]}(1 \leq k \leq n-1)$, and $\sum_{i=1}^{n} x_{[i]}=\sum_{i=1}^{n} y_{[i]}$, then $x$ is said to be majorized by $y$, denote it by $x \prec y$.

Definition 1.2. $A \subseteq R^{n}$ is called symmetric set, if $x \in A$ implies $P x \in A$ for $n \times n$ permutation matrix $P$.

Definition 1.3. $f: A \rightarrow R\left(A \subset R^{n}\right)$ is called Schur-convex if $x \prec y$, then

$$
f(x) \leq f(y) .
$$

It is called strictly Schur-convex if the inequality is strict; $f(x)$ is called Schur-concave (resp., strictly Schur-concave) if the inequality (1.8) is reversed.

Definition 1.4. $f: A \rightarrow R$ is called symmetric if for every permutation matrix $P$,

$$
f(P x)=f(x)
$$

for all $x \in A$.

Let the mark " $x \leq y$ " stand for $x_{i} \leq y_{i}, i=1,2, \ldots, n$.

Definition 1.5. $f: A\left(\subseteq R^{n}\right) \rightarrow R$ is called monotonic increasing function if $x \leq y$, then $f(x) \leq f(y)$.

In this paper, we prove the functions $c_{r}(x)$ and $c_{r}(x) / c_{r-1}(x)$ to be Schur-convex functions in $R_{+}^{n}=\left\{\left(x_{1}, x_{2}, \ldots, x_{n}\right) \mid x_{i}>0, i=1,2, \ldots, n\right\}$. Some inequalities for them are established by using of the theory of majorization. "Ky Fan" inequality is generalized. We show that inequality (1.6) is true for $n>2$, and thus the problem in [7] is solved.

\section{Lemma}

In this section, We give the following lemmas for the proofs of our main results. Every Schur-convex function is a symmetric function [11]. It is not hard to see that not every 
symmetric function can be a Schur-convex function [9, page 258]. However, we have the following so-called Schur's condition.

Lemma 2.1 [9, page 259]. Let $f(x)=f\left(x_{1}, x_{2}, \ldots, x_{n}\right)$ be symmetric and have continuous partial derivative on $I^{n}=I \times I \times \cdots \times I$ ( $n$ copies), where $I$ is an open interval. Then $f$ : $I^{n} \rightarrow R$ is Schur-convex if and only if

$$
\left(x_{i}-x_{j}\right)\left(\frac{\partial f}{\partial x_{i}}-\frac{\partial f}{\partial x_{j}}\right) \geq 0
$$

on $I^{n}$. It is strictly Schur-convex if (2.1) is a strict inequality for $x_{i} \neq x_{j}, 1 \leq i, j \leq n$.

In Schur's condition, the domain of $f(x)$ does not have to be a Cartesian product $I^{n}$. Lemma 2.1 remains true if we replace $I^{n}$ by a set $A \subseteq R^{n}$ with the following properties ([6, page 57]):

(i) $A$ is convex and has a nonempty interior,

(ii) $A$ is symmetric.

Lemma 2.2 [10]. Suppose that $x_{i}>0, i=1,2, \ldots, n, \sum_{i=1}^{n} x_{i}=s, c \geq s$, then

$$
\frac{c-x}{n c / s-1}=\left(\frac{c-x_{1}}{n c / s-1}, \ldots, \frac{c-x_{n}}{n c / s-1}\right) \prec\left(x_{1}, x_{2}, \ldots, x_{n}\right)=x .
$$

Lemma 2.3 [10]. Suppose that $x_{i}>0, i=1,2, \ldots, n, \sum_{i=1}^{n} x_{i}=s, c \geq s$, then

$$
\frac{c+x}{s+n c}=\left(\frac{c+x_{1}}{s+n c}, \frac{c+x_{2}}{s+n c}, \ldots, \frac{c+x_{n}}{s+n c}\right) \prec\left(\frac{x_{1}}{s}, \frac{x_{2}}{s}, \ldots, \frac{x_{n}}{s}\right)=\frac{x}{s} .
$$

Lemma 2.4 [6]. Suppose that $x_{i}>0, i=1,2, \ldots, n, \sum_{i=1}^{n} x_{i}=s$, then

$$
\frac{s}{n}=\left(\frac{s}{n}, \frac{s}{n}, \ldots, \frac{s}{n}\right) \prec\left(x_{1}, x_{2}, \ldots, x_{n}\right)=x .
$$

Lemma 2.5. Suppose that $x_{i}>0, i=1,2, \ldots, n$. Let

$$
\bar{x}_{i}=\left(x_{1}, \ldots, x_{i-1}, x_{i+1}, \ldots, x_{n}\right) .
$$

Then we have

$$
c_{r}(x)=x_{i} c_{r-1}(x)+c_{r}\left(\bar{x}_{i}\right) .
$$

Proof. It is easy to see that

$$
\begin{gathered}
c_{r}(x)=\sum_{i_{1}+i_{2}+\cdots+i_{n}=r} x_{i}^{i_{1}} \cdots x_{n}^{i_{n}}=x_{i}^{r}+x_{i}^{r-1} c_{1}\left(\bar{x}_{i}\right)+\cdots+c_{r}\left(\bar{x}_{i}\right), \\
c_{r-1}(x)=x_{i}^{r-1}+x_{i}^{r-2} c_{1}\left(\bar{x}_{i}\right)+\cdots+c_{r-1}\left(\bar{x}_{i}\right) .
\end{gathered}
$$

Hence

$$
c_{r}(x)=x_{i} c_{r-1}(x)+c_{r}\left(\bar{x}_{i}\right)
$$


4 The complete elementary symmetric function

Lemma 2.6 [3]. Suppose that $a=\left(a_{1}, a_{2}, \ldots, a_{n}\right), a_{i} \geq 0, i=1,2, \ldots, n$, and that $r \geq 1$ is an integer, then

$$
D_{r}^{2}(a) \leq D_{r-1}(a) D_{r+1}(a)
$$

\section{Main results}

In this section we give our main results. Some Schur-convex functions of the complete elementary symmetric function are given here. Some analytic inequalities are established. THEOREM 3.1. The complete elementary symmetric function

$$
c_{r}=c_{r}(x)=C_{n}^{[r]}(x)=\sum_{i_{1}+\cdots+i_{n}=r} x_{1}^{i_{1}} \cdots x_{n}^{i_{n}}
$$

is a Schur-convex function in $R_{+}^{n}$, and is increasing in $x_{i}, i=1,2, \ldots, n$.

Proof. In the first, we prove that $c_{r}(x)$ is an increasing function with respect to $x_{i}$. In fact, by Lemma 2.5 , we have

$$
\frac{\partial c_{r}(x)}{\partial x_{i}}=c_{r-1}(x)+x_{i} \frac{\partial c_{r-1}(x)}{\partial x_{i}}
$$

We can inductively conclude that

$$
\frac{\partial c_{r}(x)}{\partial x_{i}} \geq 0, \quad i=1,2, \ldots, n
$$

Hence, $c_{r}(x)$ is an increasing function in $x_{i}$.

Next, we prove that $c_{r}(x)$ is a Schur-convex function in $R_{+}^{n}$. It is clear that $c_{r}(x)$ is symmetric and have continuous partial derivatives in $R_{+}^{n}$. By Lemma 2.1, we only need prove that

$$
\left(x_{i}-x_{j}\right)\left(\frac{\partial c_{r}(x)}{\partial x_{i}}-\frac{\partial c_{r}(x)}{\partial x_{j}}\right) \geq 0, \quad i \neq j .
$$

This can be obtained by induction.

(i) When $r=2$, differentiating $c_{r}(x)$ with respect to $x_{i}$, we obtain

$$
\frac{\partial c_{r}(x)}{\partial x_{i}}=c_{r-1}(x)+x_{i} \frac{\partial c_{r-1}(x)}{\partial x_{i}}=\sum_{k=1}^{n} x_{k}+x_{i}
$$

And so

$$
\left(x_{i}-x_{j}\right)\left(\frac{\partial c_{r}(x)}{\partial x_{i}}-\frac{\partial c_{r}(x)}{\partial x_{j}}\right)=\left(x_{i}-x_{j}\right)^{2} \geq 0 .
$$

(ii) Assume that (3.4) is true for $r-1$. Then, still by Lemma 2.5, it follows that

$$
\frac{\partial c_{r}(x)}{\partial x_{i}}=c_{r-1}(x)+x_{i} \frac{\partial c_{r-1}(x)}{\partial x_{i}}, \quad \frac{\partial c_{r}(x)}{\partial x_{j}}=c_{r-1}(x)+x_{j} \frac{\partial c_{r-1}(x)}{\partial x_{j}}
$$


Noticing

$$
\begin{aligned}
\frac{\partial c_{r}(x)}{\partial x_{i}}-\frac{\partial c_{r}(x)}{\partial x_{j}} & =x_{i} \frac{\partial c_{r-1}(x)}{\partial x_{i}}-x_{j} \frac{\partial c_{r-1}(x)}{\partial x_{j}} \\
& =x_{i} \frac{\partial c_{r-1}(x)}{\partial x_{i}}-x_{j} \frac{\partial c_{r-1}(x)}{\partial x_{i}}+x_{j} \frac{\partial c_{r-1}(x)}{\partial x_{i}}-x_{j} \frac{\partial c_{r-1}(x)}{\partial x_{j}} \\
& =\left(x_{i}-x_{j}\right) \frac{\partial c_{r-1}(x)}{\partial x_{i}}+x_{j}\left(\frac{\partial c_{r-1}(x)}{\partial x_{i}}-\frac{\partial c_{r-1}(x)}{\partial x_{j}}\right)
\end{aligned}
$$

we get

$$
\begin{aligned}
& \left(x_{i}-x_{j}\right)\left(\frac{\partial c_{r}(x)}{\partial x_{i}}-\frac{\partial c_{r}(x)}{\partial x_{j}}\right) \\
& \quad=\left(x_{i}-x_{j}\right)^{2} \frac{\partial c_{r-1}(x)}{\partial x_{i}}+x_{j}\left(x_{i}-x_{j}\right)\left(\frac{\partial c_{r-1}(x)}{\partial x_{i}}-\frac{\partial c_{r-1}(x)}{\partial x_{j}}\right) \geq 0
\end{aligned}
$$

From (i) and (ii), by mathematical induction method, inequality (3.4) is true. Thus, the proof is complete.

Theorem 3.2. The function $\phi_{r}(x)=c_{r}(x) / c_{r-1}(x)$ is a Schur-convex function in $R_{+}^{n}$, and is increasing in $x_{i}, i=1,2, \ldots, n$, where $r \geq 1$ is a positive integer.

Proof. It is clear that $\phi_{r}(x)$ is symmetric and have continuous partial derivatives in $R_{+}^{n}$. Differentiating $\phi_{r}(x)$ with respect to $x_{i}$, we have

$$
\frac{\partial \phi_{r}(x)}{\partial x_{i}}=\frac{1}{\left(c_{r-1}(x)\right)^{2}}\left[c_{r-1}(x) \frac{\partial c_{r}(x)}{\partial x_{i}}-c_{r}(x) \frac{\partial c_{r-1}(x)}{\partial x_{i}}\right]
$$

By Lemma 2.5 and computing, we derive

$$
\frac{\partial \phi_{r}(x)}{\partial x_{i}}-\frac{\partial \phi_{r}(x)}{\partial x_{j}}=\frac{1}{\left(c_{r-1}(x)\right)^{2}}\left[c_{r}\left(\bar{x}_{j}\right) \frac{\partial c_{r-1}(x)}{\partial x_{j}}-c_{r}\left(\bar{x}_{i}\right) \frac{\partial c_{r-1}(x)}{\partial x_{i}}\right]
$$

Notice

$$
\begin{aligned}
\frac{\partial c_{r}(x)}{\partial x_{i}} & =c_{r-1}(x)+x_{i} \frac{\partial c_{r-1}(x)}{\partial x_{i}}=c_{r-1}(x)+x_{i}\left[c_{r-2}(x)+x_{i} \frac{\partial c_{r-2}(x)}{\partial x_{i}}\right] \\
& =c_{r-1}(x)+x_{i} c_{r-2}(x)+x_{i}^{2} \frac{\partial c_{r-2}(x)}{\partial x_{i}}=\cdots \\
& =c_{r-1}(x)+x_{i} c_{r-2}(x)+x_{i}^{2} c_{r-3}(x)+\cdots+x_{i}^{r-2} c_{1}(x)+x_{i}^{r-1} .
\end{aligned}
$$


6 The complete elementary symmetric function

By Lemma 2.5 and using (3.12), we have

$$
\begin{aligned}
& \frac{\partial \phi_{r}(x)}{\partial x_{i}}=\left(c_{r-1}(x) c_{r-1}(x)-c_{r}(x) c_{r-2}(x)\right)+x_{i}\left(c_{r-1}(x) c_{r-2}(x)-c_{r}(x) c_{r-3}(x)\right) \\
& +\cdots+x_{i}^{r-2}\left(c_{r-1}(x) c_{1}(x)-c_{r}(x) c_{0}(x)\right)+c_{r-1}(x) x_{i}^{r-1}, \\
& \frac{\partial \phi_{r}(x)}{\partial x_{i}}-\frac{\partial \phi_{r}(x)}{\partial x_{j}} \\
& =\frac{1}{\left(c_{r-1}(x)\right)^{2}}\left\{\left[c_{r}(x)-x_{j} c_{r-1}(x)\right]\right. \\
& \times\left[c_{r-2}(x)+x_{j} c_{r-3}(x)+x_{j}^{2} c_{r-4}(x)+\cdots+x_{j}^{r-3} c_{1}(x)+x_{i}^{r-2}\right] \\
& -\left[c_{r}(x)-x_{i} c_{r-1}(x)\right]\left[c_{r-2}(x)+x_{i} c_{r-3}(x)+x_{i}^{2} c_{r-4}(x)\right. \\
& \left.\left.+\cdots+x_{i}^{r-3} c_{1}(x)+x_{i}^{r-2}\right]\right\} \\
& =\frac{1}{\left(c_{r-1}(x)\right)^{2}}\left\{\left[c_{r-1}(x) c_{r-2}(x)-c_{r}(x) c_{r-3}(x)\right]\left(x_{i}-x_{j}\right)\right. \\
& +\left[c_{r-1}(x) c_{r-3}(x)-c_{r}(x) c_{r-4}(x)\right]\left(x_{i}^{2}-x_{j}^{2}\right)+\cdots \\
& +\left[c_{r-1}(x) c_{1}(x)-c_{r}(x) c_{0}(x)\right]\left(x_{i}^{r-2}-x_{j}^{r-2}\right) \\
& \left.+c_{r-1}(x)\left(x_{i}^{r-1}-x_{j}^{r-1}\right)\right\} .
\end{aligned}
$$

From (1.4), we obtain

$$
\frac{c_{r-1}(x)}{c_{r}(x)}>\frac{c_{r-3}(x)}{c_{r-2}(x)}, \frac{c_{r-1}(x)}{c_{r}(x)}>\frac{c_{r-4}(x)}{c_{r-3}(x)}, \ldots, \frac{c_{r-1}(x)}{c_{r}(x)}>\frac{c_{0}(x)}{c_{1}(x)} .
$$

Therefore

$$
\frac{\partial \phi_{r}(x)}{\partial x_{i}} \geq 0
$$

which means that $\phi_{r}(x)$ is increasing with respect to $x_{i}$.

Notice

$$
\left(x_{i}-x_{j}\right)\left(x_{i}^{k}-x_{j}^{k}\right) \geq 0(1 \leq k \leq r-1) .
$$

From (3.15) and (3.17), we get

$$
\left(x_{i}-x_{j}\right)\left(\frac{\partial \phi_{r}(x)}{\partial \phi_{x_{i}}}-\frac{\partial \phi_{r}(x)}{\partial \phi_{x_{j}}}\right) \geq 0 .
$$

By Lemma 2.1, $\phi_{r}(x)$ is Schur-convex in $R_{+}^{n}$. 
Theorem 3.3. Suppose that $x_{i}>0, i=1,2, \ldots, n, \sum_{i=1}^{n} x_{i}=s, c \geq s$. Then the following statements are valid:

(i)

$$
\frac{x_{1}+x_{2}+\cdots+x_{n}}{n} \leq\left(D_{r}(x)\right)^{1 / r}
$$

(ii)

$$
\frac{c_{r}(c-x)}{c_{r}(x)} \leq\left(\frac{n c}{s}-1\right) \frac{c_{r-1}(c-x)}{c_{r-1}(x)}
$$

Proof. (i) By Theorem 3.1 and Lemma 2.4, we have $c_{r}(s / n) \leq c_{r}(x)$. From this, we obtain (3.19).

(ii) By Theorem 3.2 and Lemma 2.2, we have $\phi_{r}((c-x) /(n c / s-1)) \leq \phi_{r}(x)$, which shows that (3.20) is true.

Theorem 3.4. Suppose that $x_{i}>0, i=1,2, \ldots, n$, and $\sum_{i=1}^{n} x_{i}=s, c>0$, then

$$
\frac{c_{r}(c+x)}{c_{r}(x)} \leq\left(\frac{n c}{s}+1\right) \frac{c_{r-1}(c+x)}{c_{r-1}(x)}
$$

Proof. By Theorem 3.2 and Lemma 2.3, we have $\phi_{r}((c+x) /(s+n c)) \leq \phi_{r}(x / s)$, from which we obtain (3.21).

Using Theorems 3.3 and 3.4, we can immediately get the following consequences.

Corollary 3.5. Suppose that $x_{i}>0, \sum_{i=1}^{n} x_{i}=s, c \geq s$, then

$$
\begin{aligned}
\frac{c_{r}(c-x)}{c_{r}(x)} & \leq\left(\frac{n c}{s}-1\right) \frac{c_{r-1}(c-x)}{c_{r-1}(x)} \leq\left(\frac{n c}{s}-1\right)^{2} \frac{c_{r-2}(c-x)}{c_{r-2}(x)} \\
& \leq \cdots \leq\left(\frac{n c}{s}-1\right)^{r} \frac{c_{0}(c-x)}{c_{0}(x)}=\left(\frac{n c}{s}-1\right)^{r} .
\end{aligned}
$$

Remark 3.6. Let $c=1$, we can establish the converse inequality of "Ky Fan" inequality [1], that is

$$
\frac{\sum_{i=1}^{n} x_{i}}{\sum_{i=1}^{n}\left(1-x_{i}\right)} \leq\left(\frac{c_{r}(x)}{c_{r}(1-x)}\right)^{1 / r} .
$$

Corollary 3.7. Suppose that $x_{i}>0, \sum_{i=1}^{n} x_{i}=s, c \geq 0$, then

$$
\begin{aligned}
\frac{c_{r}(c+x)}{c_{r}(x)} & \leq\left(\frac{n c}{s}+1\right) \frac{c_{r-1}(c+x)}{c_{r-1}(x)} \leq\left(\frac{n c}{s}+1\right)^{2} \frac{c_{r-2}(c+x)}{c_{r-2}(x)} \\
& \leq \cdots \leq\left(\frac{n c}{s}+1\right)^{r} \frac{c_{0}(c-x)}{c_{0}(x)}=\left(\frac{n c}{s}+1\right)^{r} .
\end{aligned}
$$


8 The complete elementary symmetric function

Theorem 3.8. Suppose that $0<x_{i} \leq 1 / 2, i=1,2, \ldots, n$, let $1-x=\left(1-x_{1}, 1-x_{2}, \ldots, 1-\right.$ $\left.x_{n}\right)$, then

$$
\frac{c_{n}(1-x)}{c_{n}(x)} \geq \cdots \geq \frac{c_{r}(1-x)}{c_{r}(x)} \geq \frac{c_{r-1}(1-x)}{c_{r-1}(x)} \geq \cdots \geq \frac{c_{1}(1-x)}{c_{1}(x)}=\frac{A_{n}(1-x)}{A_{n}(x)},
$$

where $A_{n}(x)$ is arithmetic mean of real numbers $x_{1}, x_{2}, \ldots, x_{n}$.

Proof. By Theorem 3.2, $\phi_{r}(x)=c_{r}(x) / c_{r-1}(x)$ is an increasing function in $A=\left\{\left(x_{1}, x_{2}, \ldots\right.\right.$, $\left.\left.x_{n}\right) \mid 0<x_{i}<1\right\}$, and $1-x \geq x$. Therefore

$$
\phi_{r}(1-x) \geq \phi_{r}(x)
$$

Or

$$
\frac{c_{r}(1-x)}{c_{r-1}(1-x)} \geq \frac{c_{r}(x)}{c_{r-1}(x)}
$$

It means (3.25) is valid.

Remark 3.9. The inequality (3.25) is of the type of the "Ky Fan" inequality [1]:

$$
\frac{G_{n}(1-x)}{G_{n}(x)} \geq \frac{A_{n}(1-x)}{A_{n}(x)} .
$$

Theorem 3.10. Suppose that $x_{i}>0, i=1,2, \ldots, n, n \geq 2$, then

$$
D_{r-2}(x) D_{r+2}(x)-D_{r-1}(x) D_{r+1}(x) \geq 0 .
$$

Proof. By Lemma 2.6, we can obtain that

$$
D_{r}^{2}(x) \leq D_{r-1}(x) D_{r+1}(x) ; \quad D_{r-1}^{2}(x) \leq D_{r-2}(x) D_{r}(x) ; \quad D_{r+1}^{2}(x) \leq D_{r}(x) D_{r+2}(x)
$$

From them, it follows that

$$
D_{r-2}(x) D_{r+2}(x)-D_{r-1}(x) D_{r+1}(x) \geq 0 .
$$

Remark 3.11. Theorem 3.10 shows the inequality (1.6) is true for $n>2$. So, our result solve the problem given by Menon in [7].

\section{Acknowledgments}

The author is greatly indebted to the referees for their valuable suggestions and comments. A project supported by Scientific Research Fund of Hunan Provincial Education Department (China) (granted 03C427). 


\section{References}

[1] E. F. Beckenbach and R. Bellman, Inequalities, Ergebnisse der Mathematik und ihrer Grenzgebiete, N. F., vol. 30, Springer, Berlin, 1961.

[2] D. W. Detemple and J. M. Robertson, On generalized symmetric means of two variables, Univerzitet u Beogradu. Publikacije Elektrotehničkog Fakulteta. Serija Matematika i Fizika (1979), no. 634-677, 236-238.

[3] K. Guan, Inequalities of generalized $k$-order symmetric mean, Journal of Chongqing Teachers College (Natural Science Edition) 15 (1998), no. 3, 40-43 (chinese).

[4] G. H. Hardy, J. E. Littlewood, and G. Pólya, Some simple inequalities satisfied by convex functions, Messenger of Mathematics 58 (1929), 145-152.

[5] J. C. Kuang, Applied Inequalities, 2nd ed., Human education Press, Changsha, 1993.

[6] A. W. Marshall and I. Olkin, Inequalities: Theory of Majorization and Its Applications, Mathematics in Science and Engineering, vol. 143, Academic Press, New York, 1979.

[7] K. V. Menon, Inequalities for symmetric functions, Duke Mathematical Journal 35 (1968), 37-45.

[8] D. S. Mitrinović, Analytic Inequalities, Die Grundlehren der mathematischen Wisenschaften, vol. 1965, Springer, New York, 1970.

[9] A. W. Roberts and D. E. Varberg, Convex Functions, Pure and Applied Mathematics, vol. 57, Academic Press, New York, 1973.

[10] H. N. Shi, Refinement and generalization of a class of inequalities for symmetric functions, Mathematics in Practice and Theory 29 (1999), no. 4, 81-84 (Chinese).

[11] X.-M. Zhang, Optimization of Schur-convex functions, Mathematical Inequalities \& Applications 1 (1998), no. 3, 319-330.

Kaizhong Guan: Department of Mathematics and Physics, Nanhua University, Hengyang,

Hunan 421001, China

E-mail address: kaizhongguan@yahoo.com.cn 